\title{
Design of a thermal imaging diagnostic using 90-degree, off-axis parabolic mirrors
}

\author{
Robert M. Malone* ${ }^{\mathrm{a}}$, Steven A. Becker ${ }^{\mathrm{b}}$, Daniel H. Dolan ${ }^{\mathrm{c}}$, Richard G. Hacking ${ }^{\mathrm{a}}$, \\ Randy J. Hickman ${ }^{c}$, Morris I. Kaufman ${ }^{\text {a }}$, Gerald D. Stevens ${ }^{\mathrm{d}}$, William D. Turley ${ }^{\mathrm{d}}$ \\ ${ }^{a}$ NSTec, Los Alamos Operations, 182 East Gate Drive, Los Alamos, NM USA 87544; \\ ${ }^{b}$ NSTec, North Las Vegas Operations, PO Box 98521, Las Vegas, NV USA 89193; \\ 'Sandia National Laboratories, PO Box 5800, Albuquerque, NM USA 87185; \\ ${ }^{\mathrm{d}}$ NSTec, Special Technologies Laboratory, 5540 Ekwill St., Santa Barbara, CA USA 93111
}

\begin{abstract}
Thermal imaging is an important, though challenging, diagnostic for shockwave experiments. Shock-compressed materials undergo transient temperature changes that cannot be recorded with standard (greater than ms response time) infrared detectors. A further complication arises when optical elements near the experiment are destroyed. We have designed a thermal-imaging system for studying shock temperatures produced inside a gas gun at Sandia National Laboratories. Inexpensive, diamond-turned, parabolic mirrors relay an image of the shocked target to the exterior of the gas gun chamber through a sapphire vacuum port. The 3000-5000-nm portion of this image is directed to an infrared camera which acquires a snapshot of the target with a minimum exposure time of 150 ns. A special mask is inserted at the last intermediate image plane, to provide dynamic thermal background recording during the event. Other wavelength bands of this image are split into high-speed detectors operating at 900-1700 nm, and at 1700-3000 nm for timeresolved pyrometry measurements. This system incorporates 90-degree, off-axis parabolic mirrors, which can collect low f/\# light over a broad spectral range, for high-speed imaging. Matched mirror pairs must be used so that aberrations cancel. To eliminate image plane tilt, proper tip-to-tip orientation of the parabolic mirrors is required. If one parabolic mirror is rotated 180 degrees about the optical axis connecting the pair of parabolic mirrors, the resulting image is tilted by 60 degrees. Different focal-length mirrors cannot be used to magnify the image without substantially sacrificing image quality. This paper analyzes performance and aberrations of this imaging diagnostic.
\end{abstract}

Keywords: mid-IR imaging, off-axis parabolic mirrors, gas gun imaging, IR camera, pyrometry

\section{INTRODUCTION}

Mirrors are a cost-effective component in broadband infrared (IR) diagnostics, particularly in harsh environments. Lensbased systems tend to be expensive (particularly at low f/\#) and can only be optimized for a narrow wavelength range. Diamond-turned mirrors, however, function over a wide spectral range, and have become competitive with lenses in both cost and quality. This paper investigates the use of diamond-turned mirrors in an IR imaging system.

A key application for mirror-based optical relay systems is IR emission measurements during plate impact studies. These experiments are performed in a light gas gun (Figure 1), which uses compressed nitrogen or helium to drive a projectile into a target of interest at velocities of 100-600 m/s. For several microseconds after impact, the target undergoes temperature rises of $100-1000{ }^{\circ} \mathrm{K}$. Fast multichannel pyrometry and/or infrared imaging may be used to interrogate the target before the ultimate destruction of the target and all optical components within the target chamber. Maximum light throughput and anticipated hardware destruction are critical to the infrared relay design. $\mathrm{A}$ replaceable $\mathrm{CaF}_{2} \mathrm{blast}$ window is placed in front of the expensive sapphire vacuum port window.

*malonerm@nv.doe.gov; phone 1-505-663-2014; fax 1-505-663-2003 


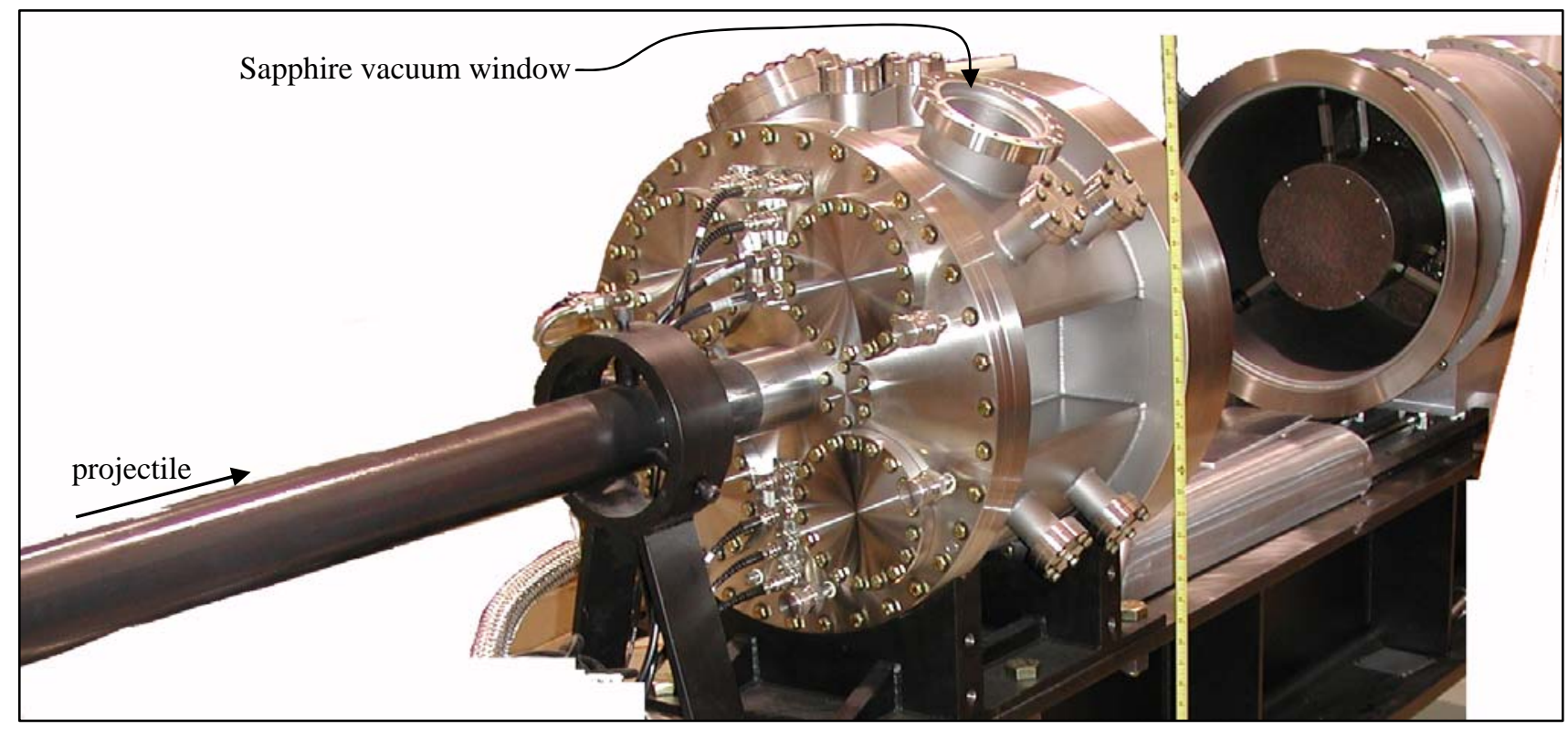

Fig. 1. Gas gun for IR imaging. The projectile enters into the vacuum target chamber from the right. There are several port openings in the target chamber for installing diagnostics. The debris catch tank has been opened on the left.

\section{OPTICAL RELAY DESIGN}

Off-axis parabolic mirrors combine the functionality of relay lenses and turning mirrors, though there is scant literature regarding the use of such mirrors in imaging applications. Figure 2 illustrates the use of off-axis parabolic mirrors

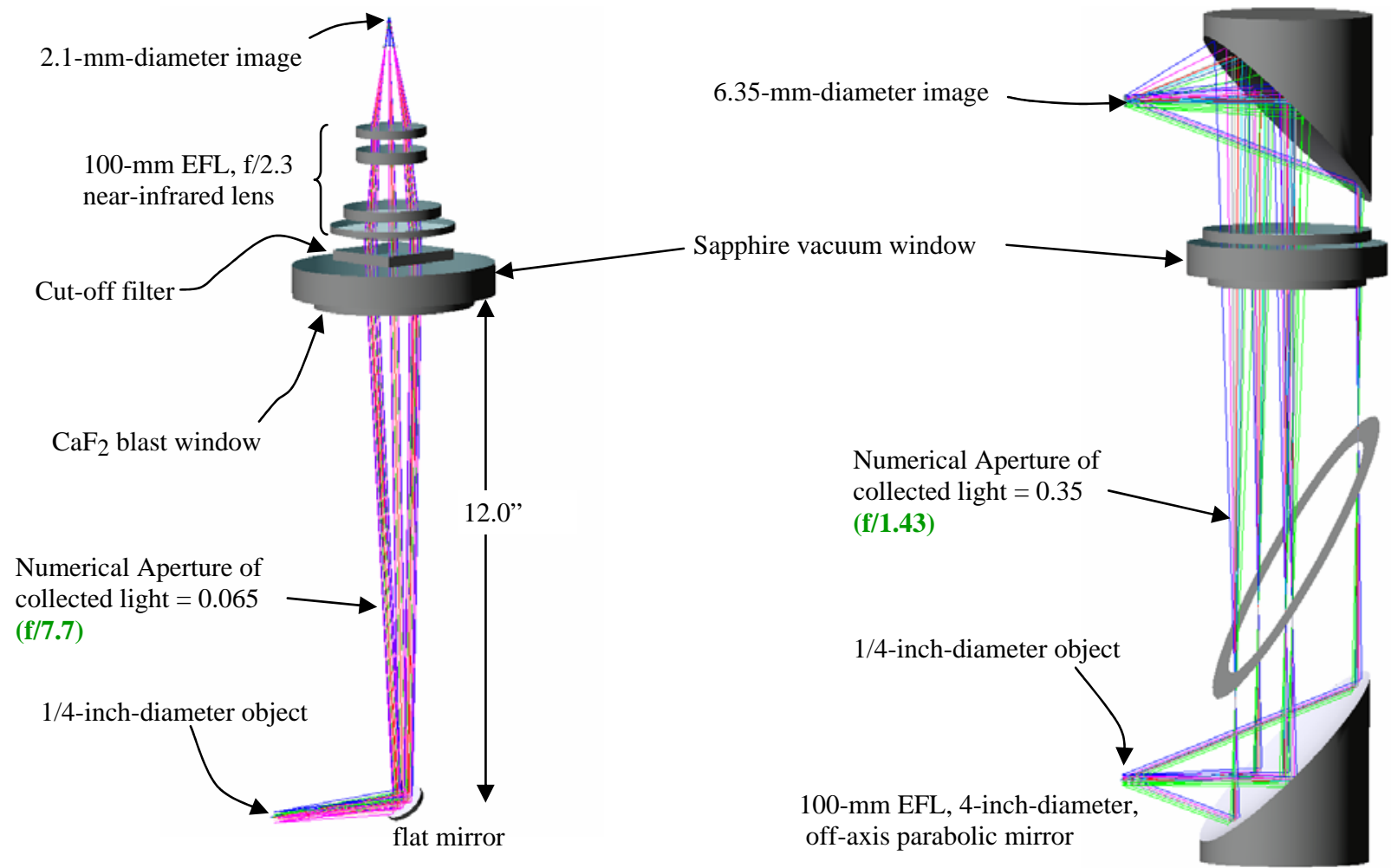

Fig. 2. Comparison of standard IR lens imaging to the 90-degree, off-axis parabolic mirror imaging. 
compared with a lens-based relay. Both systems collect telecentric light and are designed for the gas gun shown in Figure 1. We are viewing 1/4-inchdiameter areas from a target hit by the projectile. Expensive IR lenses must be placed outside the vacuum port where they will not be destroyed. Since the parabolic mirrors cost only $1 / 8$ that of the IR lens, it is feasible to locate the first mirror inside the target chamber where it is close to the target under study. An identical pair of parabolic mirrors is necessary to achieve good imaging. The mirror system thus collects approximately 29 times more light than the lens system while maintaining good image quality. Note that in the mirror system, light passing between the mirrors is nearly collimated. Collimation reduces aberrations caused by a tilted vacuum port window. Note also the unusual tilting of the stop aperture in the mirror system. The mirror system also produces a 1:1 image of the target, while the lens system has significant demagnification that limits imaging resolution. To produce a large-format image with the IR lens system, a 300-mm effective focal length (EFL) IR lens would have to replace the 100-mm EFL lens (shown on the left side of Figure 2). This would add considerable expense to the diagnostic.

A possible variation in IR imaging is to mix lenses and mirrors as shown in Figure 3. In doing so, one increases light collection and image size. However, the image plane is now tilted at 45 degrees and is thus unusable for thermal imaging cameras.

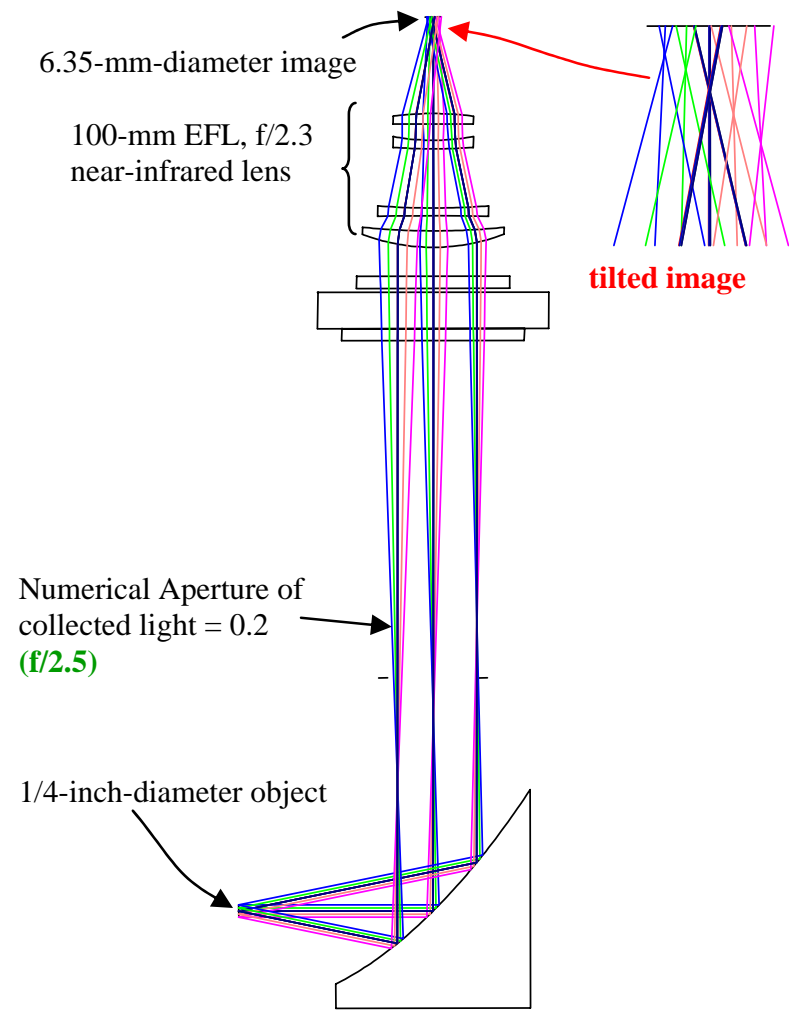

Fig. 3. Using only one parabolic mirror tilts the image.

\section{ORIENATATIONS OF 90-DEGREE, OFF-AXIS PARABOLIC MIRRORS}

A pair of off-axis parabolic mirrors may be used to eliminate tilting of the image plane, but if these mirrors have the correct rotations. Figure 4 shows a pair of 25.4-mm EFL, 1-inch-diameter, 90-degree, off-axis parabolic mirrors that have been improperly rotated. Light from a 1-mm-diameter object is telecentric at f/3. The image plane is tilted at 60 degrees. Notice that the elliptical stop is tilted by 60 degrees. The ray tracing at the image plane shows that the image quality of this tilted plane will be unacceptable for IR camera imaging. Inspection of the optical ray tracing shows that rays from a single field point map onto different regions of each parabolic mirror. To achieve the best resolution without tilting the image plane, the rays from a single field point must strike identical coordinates on each parabolic mirror. This requires the orientation of the mirrors to be "tip to tip."

Figure 5 shows proper rotation for this same pair of parabolic mirrors. The elliptical stop is still tilted by 60 degrees but there is no tilt at the image plane. Image quality is diffraction-limited.

The image quality depends on the object size. Figure 6 shows a comparison of two different object diameters being relayed. Two 150-mm EFL, 3-inch diameter, 90-degree, off-axis parabolic mirrors were used. Figure 7 shows the Modulation Transfer Function (MTF) for 1- and 6.35-mm-diameter objects. The 1-mm-diameter object has near diffraction-limited performance. Refocusing the $65-\mathrm{mm}$-diameter object by $0.4 \mathrm{~mm}$ optimized its MTF and made the image quality acceptable for the IR camera. 


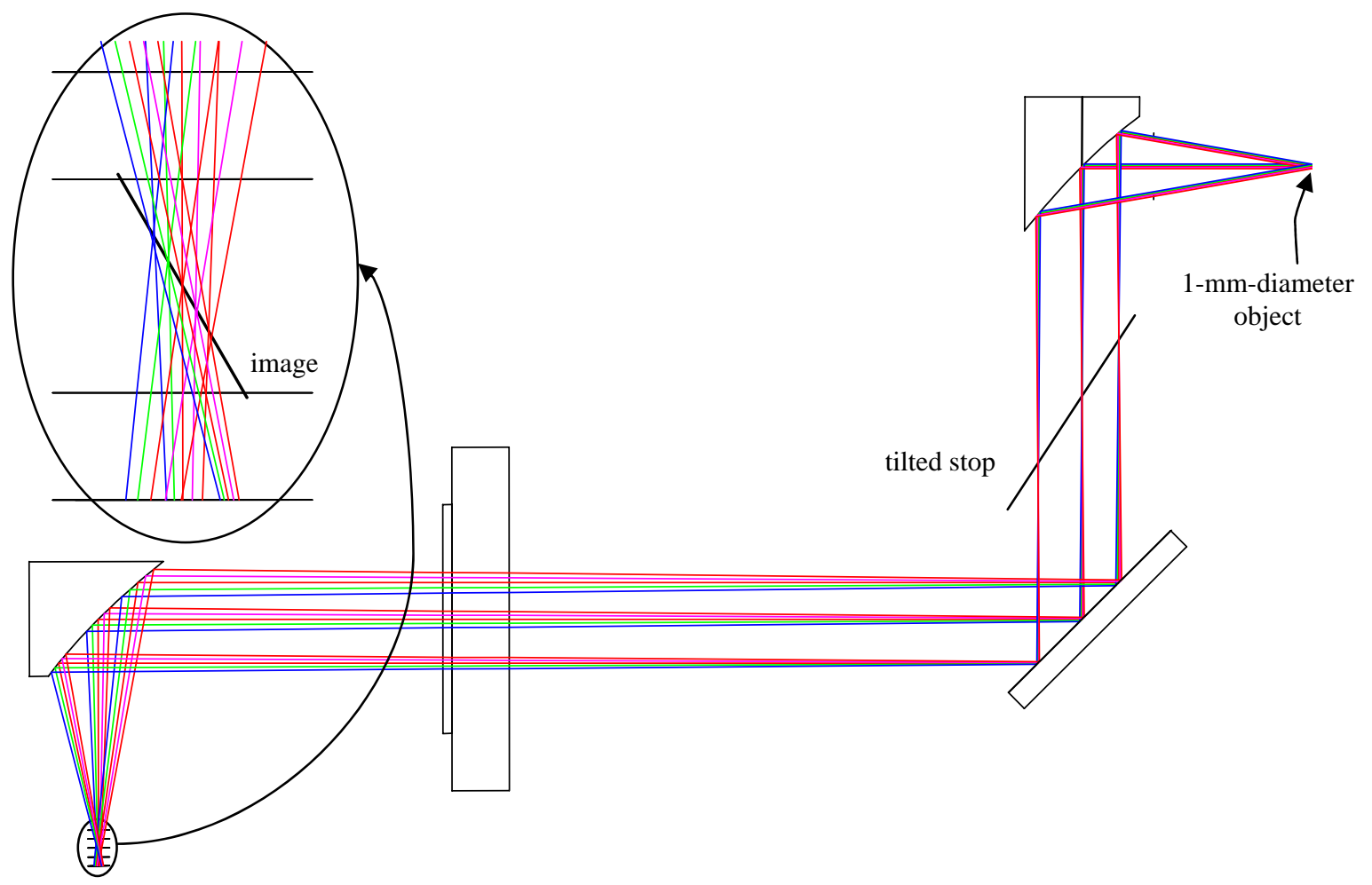

Fig. 4. The off-axis parabolic mirrors have incorrect orientations. The image plane is tilted at 60 degrees.

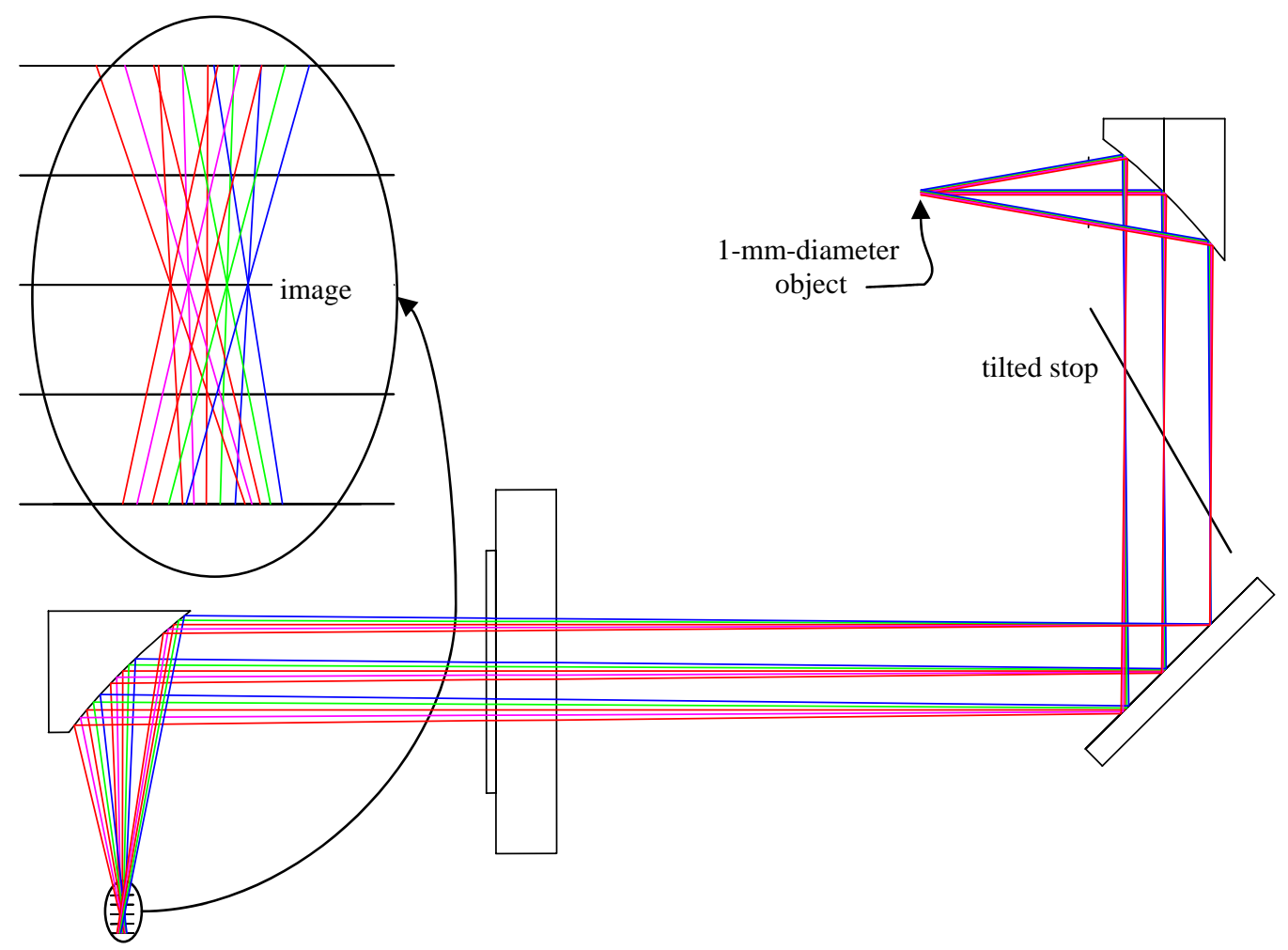

Fig. 5. The off-axis parabolic mirrors have correct orientations. The image plane is not tilted. 


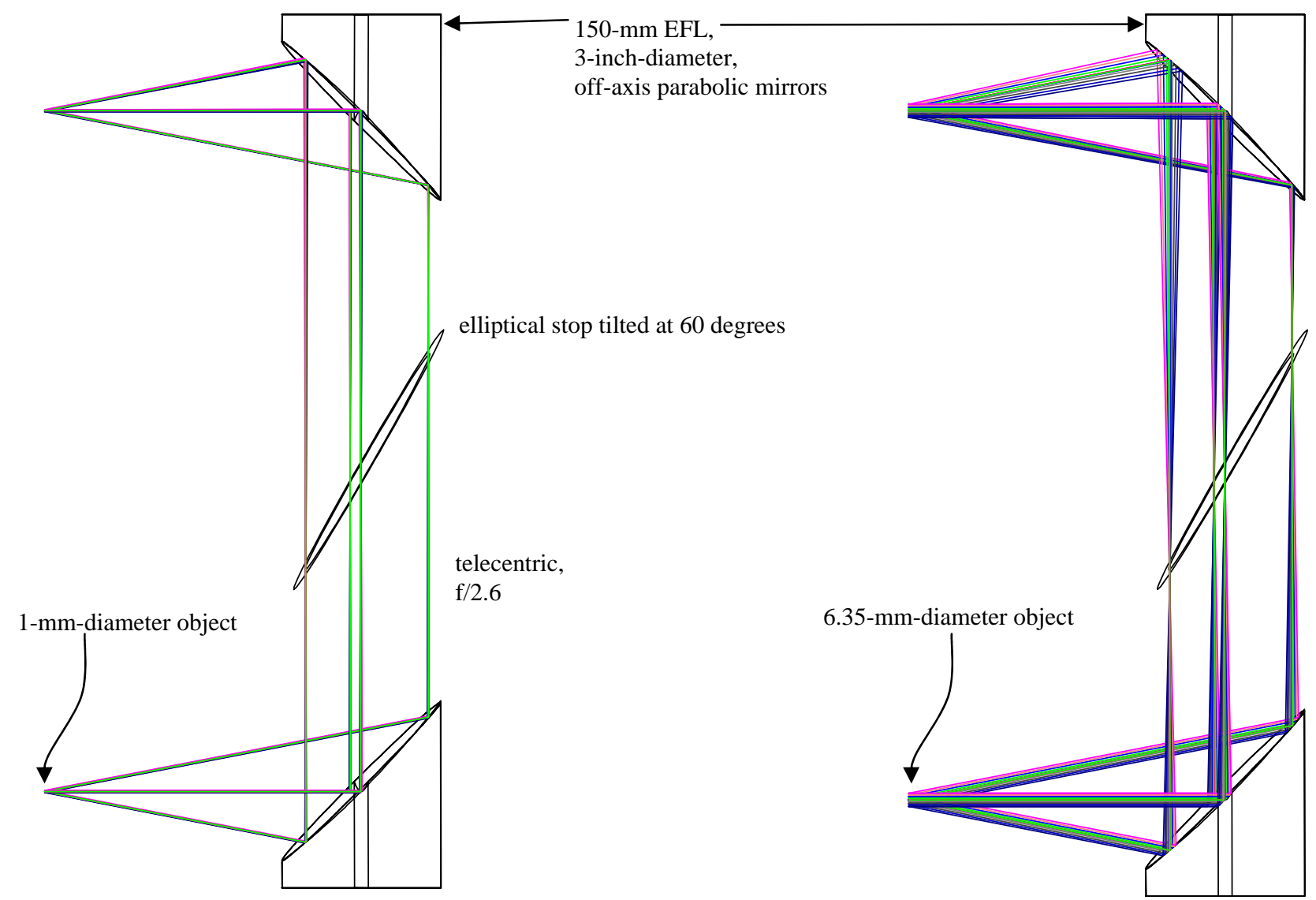

Fig 6. Ray tracing pairs of parabolic mirrors used for 1:1 imaging. All mirrors are identical. Better resolution is achieved for the 1-mm object versus the $6.35-\mathrm{mm}$ object. A $0.4-\mathrm{mm}$ defocus was applied to the $6.35-\mathrm{mm}$ image during optimization.
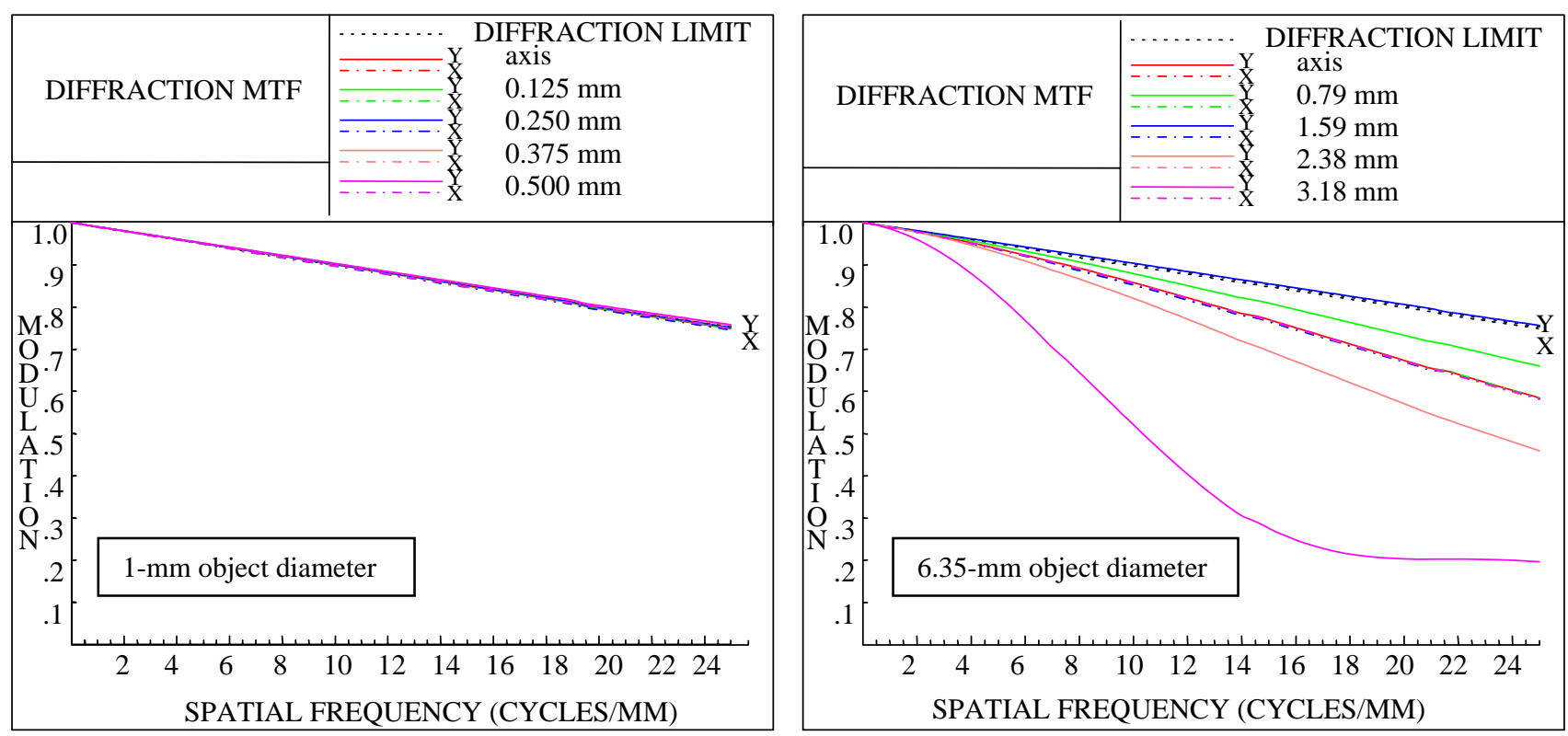

Fig. 7. Resolution depends on the object size. 
Figure 8 shows how resolution will degrade with incorrect rotation alignment of the mirrors. The alignment tolerance of two 150-mm EFL, 3-inchdiameter, 90-degree, off-axis parabolic mirrors is \pm 2 degrees. Light from a 6.35 -mm-diameter object is telecentric at $\mathrm{f} / 2.6$.

Using identical focal lengths in a 1:1 imaging configuration provides a good image. However, employing off-axis parabolic mirrors of different focal lengths does not produce imaging as good as that of optical lens systems. Figure 9 shows an example of trying to achieve a $1.5 \times$ magnification in the image by using parabolic mirrors that have focal lengths of 100 and $150 \mathrm{~mm}$. The ray tracing shows a curved image plane and the MTF shows poor image quality. The aberrations produced by the first parabolic mirror are not quite cancelled out by the second parabolic mirror. This is because the footprints of different field points map onto different curvatures on each parabolic mirror.

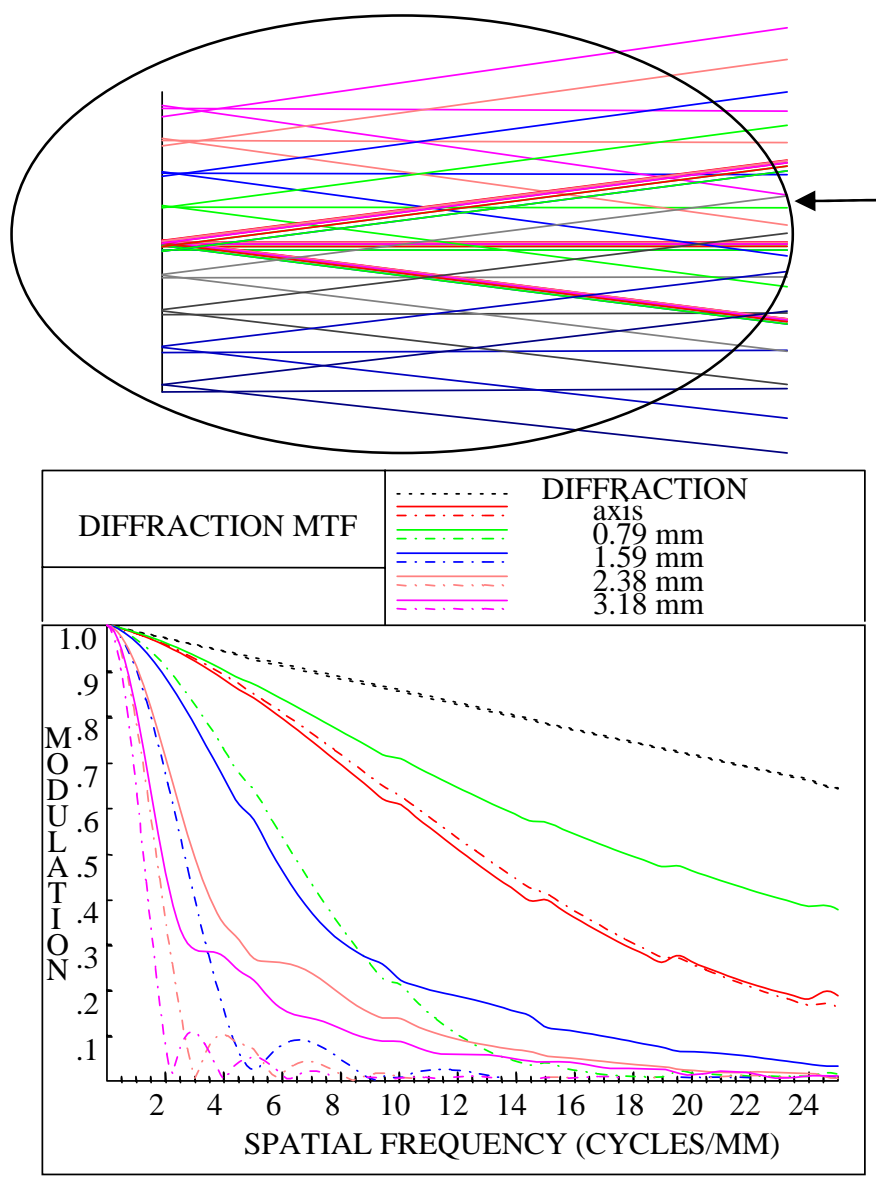

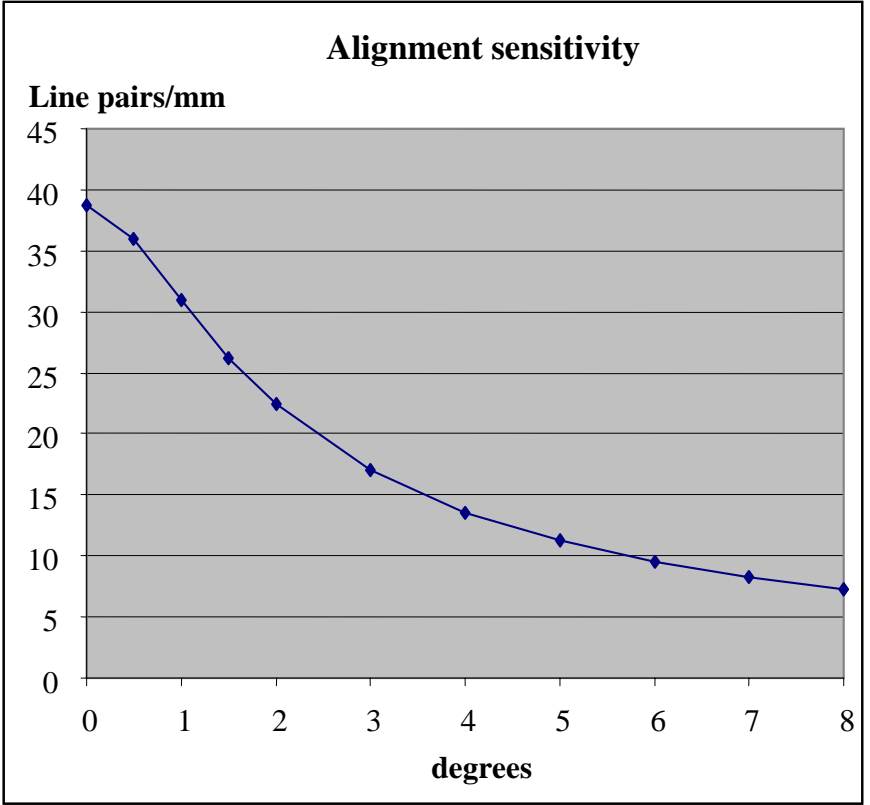

Fig 8. Alignment sensitivity for parabolic mirror rotations.

parabolic mirror, 150-mm EFL, 3-inch-diameter
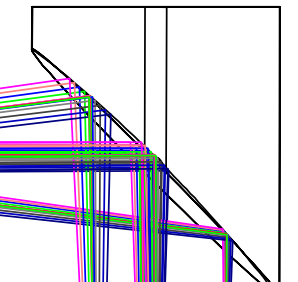

parabolic mirror, 100-mm EFL, 3-inch-diameter

Fig. 9. Different focal length, 90-degree, off-axis parabolic mirrors are used to produce $1.5 \times$ magnification. 
Anamorphic Ratio $(\mathrm{Y} / \mathrm{X})=0.99336$

Vertical FOV

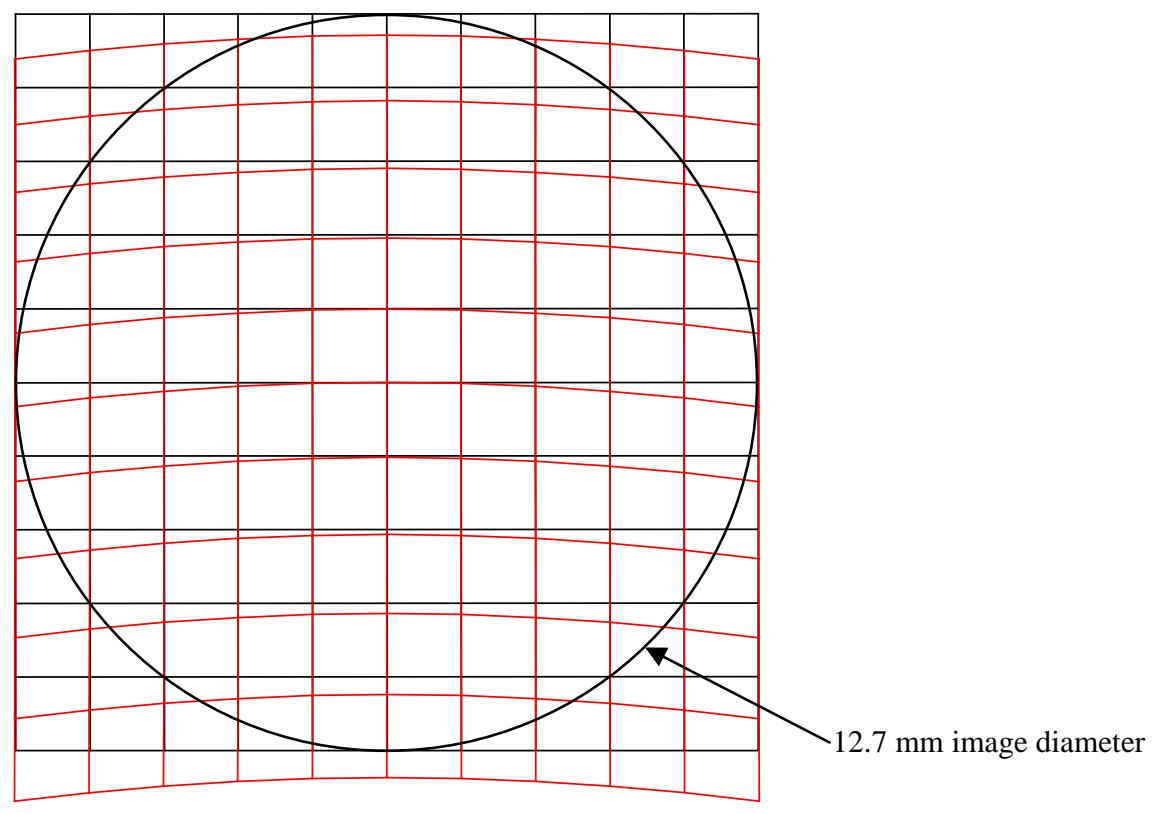

Horizontal FOV

Fig. 10. Typical distortion plot. Only about one half of the distortion can be eliminated by optimizing the air spacing between the parabolic mirrors. There is very little distortion in the $\mathrm{X}$ direction.

Even though the image quality is good for the 1:1 imaging configuration, there is considerable distortion. Figure 10 shows a typical distortion plot. Distortion remapping is performed by the computer that controls the IR camera. A special calibration procedure must be performed as part of the set up of each experiment. The amount of distortion is proportional to the focal lengths of the parabolic mirrors used.

\section{FINAL IMAGING/PYROMETRY DESIGN}

IR imaging and pyrometry for the gas gun requires the use of mirrors for broadband spectral response. The IR camera has $640 \times 512$ pixels with 20 - $\mu \mathrm{m}$ pitch, providing a $12.8-\times 10.24-\mathrm{mm}$ image size. In this application, the goal is to resolve $150 \mu \mathrm{m}$ features inside a 6.35-mm-diameter object. Thus, only 1 part in 50 across the object needs to be resolved. When using IR cameras experimenters typically record dark field images prior to the experiment and optimistically hope that thermal drift during the actual experiment will be minimal. To overcome this concern, a special mask to block a discrete portion of the image is inserted at the intermediate image plane. Thus, during the experiment, part of the image will register dark field information. This dynamic background measurement will be recorded during each experiment. Gas gun velocities range from $300-500 \mathrm{~m} / \mathrm{s}$. At the highest velocity of $500 \mathrm{~m} / \mathrm{s}$ and image integration of $500 \mathrm{~ns}$, the target will move $250 \mu \mathrm{m}$. Therefore, the depth of field should be greater than $500 \mu \mathrm{m}$ to allow for focusing errors. It would be ideal if the best focus plane is placed $125 \mu \mathrm{m}$ in front of the target. 


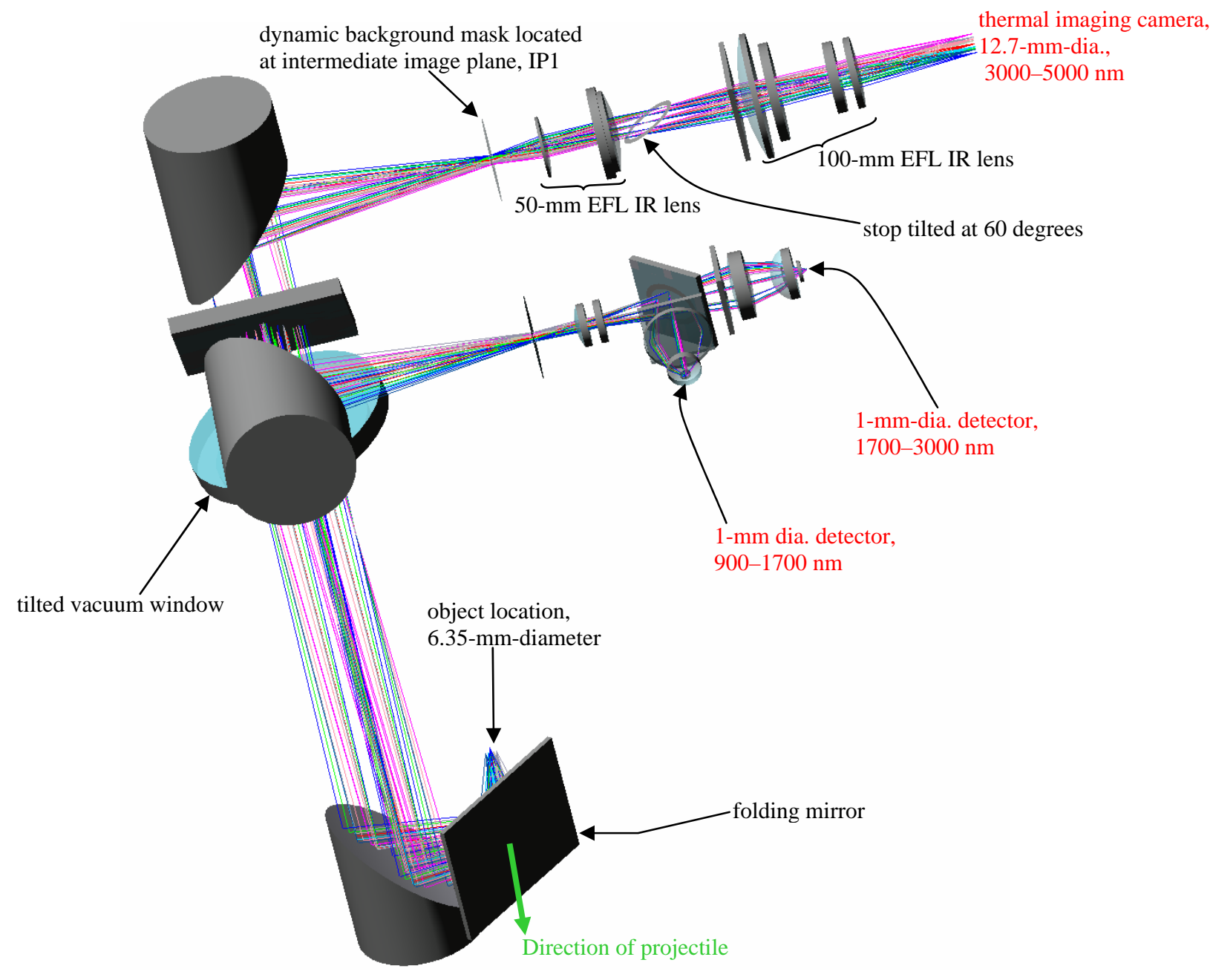

Fig. 11. 3-D perspective view of thermal imaging.

The optical design for the gas gun experiments is shown in Figure 11. The system requires a single-frame IR camera as well as two detectors that record the time history of the emissions at two different wavelength bands. Light is sent through an expensive sapphire window protected by a throw-away, thin $\mathrm{CaF}_{2}$ window. The off-axis parabolic mirror is too massive to place in the path of the projectile; a blow-away, low-mass folding mirror is used. Commercial IR camera lenses (50 and $100 \mathrm{~mm}$ EFLs) set up as a Fourier relay pair are used to magnify the intermediate image plane by $2 \times$. Custom singlet lenses, using $\mathrm{ZnSe}$ and $\mathrm{CaF}_{2}$ are used to demagnify the light by $6 \times$ onto the high-speed detectors. Filters are positioned where the light is collimated in front of the IR camera and the two detectors.

The low-mass folding mirror can be rotated 90 degrees for calibration work. Resolution and grid patterns are placed at the virtual object distance and can be back-illuminated by IR lamps. The grid is used to map the distortions discussed in the previous section and verify that there is no image tilt. This calibration exercise of pyrometry and imaging does not interfere with the projectile delivery hardware.

\section{SUMMARY}

Using parabolic mirrors in imaging applications has advantages and limitations. Using a single off-axis parabolic mirror is not useful for imaging applications because the image plane is tilted. Great care must be taken to align the pair of 
parabolic mirrors to prevent image tilting. These parabolic mirrors are useful for collecting broadband emissions due to the absence of chromatic aberrations. They can collect low f/\# light. However, the resolution at the recording camera depends on the object size, and therefore only relatively small object sizes can be imaged. There is distortion in the image which must be mapped out. The pair of off-axis parabolic mirrors must have identical focal lengths. Consequently, parabolic mirrors cannot be used to vary image magnifications.

Copyright. This manuscript has been authored by Bechtel Nevada and National Security Technologies under Contract Nos. DE-AC08-96NV11718 and DE-AC52-06NA25946 with the U.S. Department of Energy. Sandia is a multiprogram laboratory operated by Sandia Corporation, a Lockheed Martin Company, for the United States Department of Energy's National Nuclear Security Administration under contract DE-AC04-94AL85000. The United States Government retains and the publisher, by accepting the article for publication, acknowledges that the United States Government retains a nonexclusive, paid-up, irrevocable, world-wide license to publish or reproduce the published form of this manuscript, or allow others to do so, for United States Government purposes.

Disclaimer. This report was prepared as an account of work sponsored by an agency of the U.S. Government. Neither the U.S. Government nor any agency thereof, nor any of their employees, nor any of their contractors, subcontractors or their employees, makes any warranty or representation, express or implied, or assumes any legal liability or responsibility for the accuracy, completeness, or usefulness of any information, apparatus, product, or process disclosed, or represents that its use would not infringe privately own rights. Reference herein to any specific commercial product, process, or service by trade name, trademark, manufacturer, or otherwise, does not necessarily constitute or imply its endorsement, recommendation, or favoring by the U.S. Government or any agency thereof. The views and opinions of authors expressed herein do not necessarily state or reflect those of the U.S. Government or any agency thereof. 